\title{
Topographic Mapping of Motor Pools onto Skeletal Muscles
}

\author{
Michael B. Laskowski and Joshua R. Sanes \\ Department of Anatomy and Neurobiology, Washington University School of Medicine, St. Louis, Missouri 63110
}

\begin{abstract}
We have studied the segmental innervation of 2 rat skeletal muscles, the diaphragm and the serratus anterior. Both muscles are thin, flat, and composed of several sectors that form a clear rostrocaudal progression. Each is innervated through a single nerve, which is in turn supplied by motor neurons from several cervical spinal segments. Using intracellular recording, we found that in both cases, the rostrocaudal axis of the motor pool is systematically mapped onto the rostrocaudal axis of the muscle's surface. For the diaphragm, electrophysiological results were confirmed by immunohistochemical identification of denervated fibers following section of single ventral roots and by retrograde labeling of motoneurons following localized application of fluorescent dyes. In addition, an immunohistochemical method was used to study the arrangement of motor axons in the phrenic nerve, which supplies the diaphragm, and to show that contributions from individual ventral roots are compartmentalized within this nerve. We suggest that segmental ordering of axons in the nerve, axonal guidance at branch points in the nerve, and positional labels within the muscle may all contribute to the rostrocaudal mapping of motor pools onto muscle.
\end{abstract}

Topographic projections of neuronal arrays onto their targets are found in many regions of the nervous system. The most thoroughly studied of these projections is the retinotectal map (reviewed in Cowan and Hunt, 1985), but comparable maps are found in a variety of sensory systems (Somjen, 1972), including the auditory (tonotopic) and somatosensory (somatotopic) systems, as well as in the autonomic nervous system ( $\mathrm{Njå} \mathrm{and}$ Purves, 1977; Lichtman et al., 1979). Within the motor system, the arrangement of motor pools and muscles is such that the entire motor column that supplies each limb forms a roughly topographic map on that limb (Sherrington, 1892; Browne, 1950), and some individual limb muscles also receive topographically organized projections from the ventral roots and rootlets that supply them (Swett et al., 1970; Brown and Booth, 1983; Bennett and Lavides, 1984). Elucidating the rules that govern the formation of such maps has been a major goal of developmental neurobiologists. Here, we document and characterize a particularly simple and therefore potentially useful topographic map

Received Feb. 18, 1986; revised May 27, 1986; accepted June 3, 1986.

We thank J. Mosher and S. Eads for assistance, V. Friedman for illustrations, and J. Covault, J. Lichtman, and D. Purves for comments. This work was supported by grants from the Muscular Dystrophy Association and the National Institutes of Health. J.S. is an Established Investigator of the American Heart Association.

Correspondence should be addressed to Michael B. Laskowski, Department of Physiology, St. Louis University School of Medicine, 1402 South Grand Boulevard, St. Louis, Mo 63104.

Copyright (c) 1987 Society for Neuroscience $0270-6474 / 87 / 010252-09 \$ 02.00 / 0$ of neuronal connectivity: the orderly representation of the rostrocaudal axis of a single motor pool onto the rostrocaudal axis of an individual skeletal muscle.

Our interest in this projection stems from experiments in which we transplanted rat intercostal muscles from different segmental levels to a common site in the neck and studied their reinnervation by axons of the cervical sympathetic trunk. We found that muscles transplanted from rostral levels were reinnervated by a more rostrally derived subset of axons than were transplanted caudal muscles, suggesting that axons and muscles bear positionally varying labels that favor synapse formation between positionally matched partners (Wigston and Sanes, 1982, 1985). It will be difficult to learn, however, whether such a matching system might play any role in the normal development of the intercostals: Each intercostal is supplied by a separate segmental nerve, and spatial relationships of axons to individual intercostals could account for their segmentally specific innervation in normal animals. To extend our studies, we therefore sought muscles that (1) are innervated by motor axons arising from more than one spinal cord segment; (2) receive their innervation through a single nerve trunk; (3) are thin and flat, i.e., essentially 2-dimensional; and (4) have natural subdivisions or sectors that form a clear rostrocaudal progression. Two muscles meeting these criteria are the anterior serratus muscle and the diaphragm. We report here that in both muscles the rostrocaudal axis of the motor pool is mapped systematically onto the rostrocaudal axis of the muscle. In addition, for the diaphragm, we describe the segmental arrangement of axons in extra- and intramuscular portions of the motor nerve, and consider how axonal ordering could contribute to the motor pool-to-muscle map. A preliminary report of this work has appeared (Laskowski and Sanes, 1985).

\section{Materials and Methods}

Dissection. Male adult Sprague-Dawley rats (180-300 gm) were anesthetized with chloral hydrate and perfused through the left ventricle with Ringer's solution containing: $144 \mathrm{~mm} \mathrm{NaCl}, 4 \mathrm{~mm} \mathrm{KCl}, 1 \mathrm{~mm}$ $\mathrm{MgCl}_{2}, 1 \mathrm{mM} \mathrm{KH}_{2} \mathrm{PO}_{4}, 4 \mathrm{~mm}$ HEPES buffer, $11 \mathrm{~mm}$ glucose, and $2 \mathrm{mM}$ $\mathrm{CaCl}_{2}, \mathrm{pH}$ 7.3. The animal was decapitated and the upper trunk was placed in a dish with oxygenated Ringer's solution for dissection. A right dorsal laminectomy of the cervical vertebral column revealcd the spinal cord and its roots. Ventral roots were dissected with a portion of the ventral spinal cord still attached. For experiments with the serratus, roots $\mathrm{C} 6-8$ were dissected. When the diaphragm was to be studied, roots C3-6 were dissected, being careful to include the accessory phrenic nerve (Gottschall and Gruber, 1977). In both cases, the muscle of the right side was selected for study. The final preparation contained the muscle, its nerve trunk, and all of its innervating cervical roots, in continuity and free of the remainder of the animal (Fig. 1, B, C). This preparation was placed in a Sylgard-coated tissue culture dish, where it was continuously superfused with oxygenated Ringer's.

For the serratus anterior, the sixth cervical root was divided along natural subdivisions into rostral, intermediate, and caudal components. 


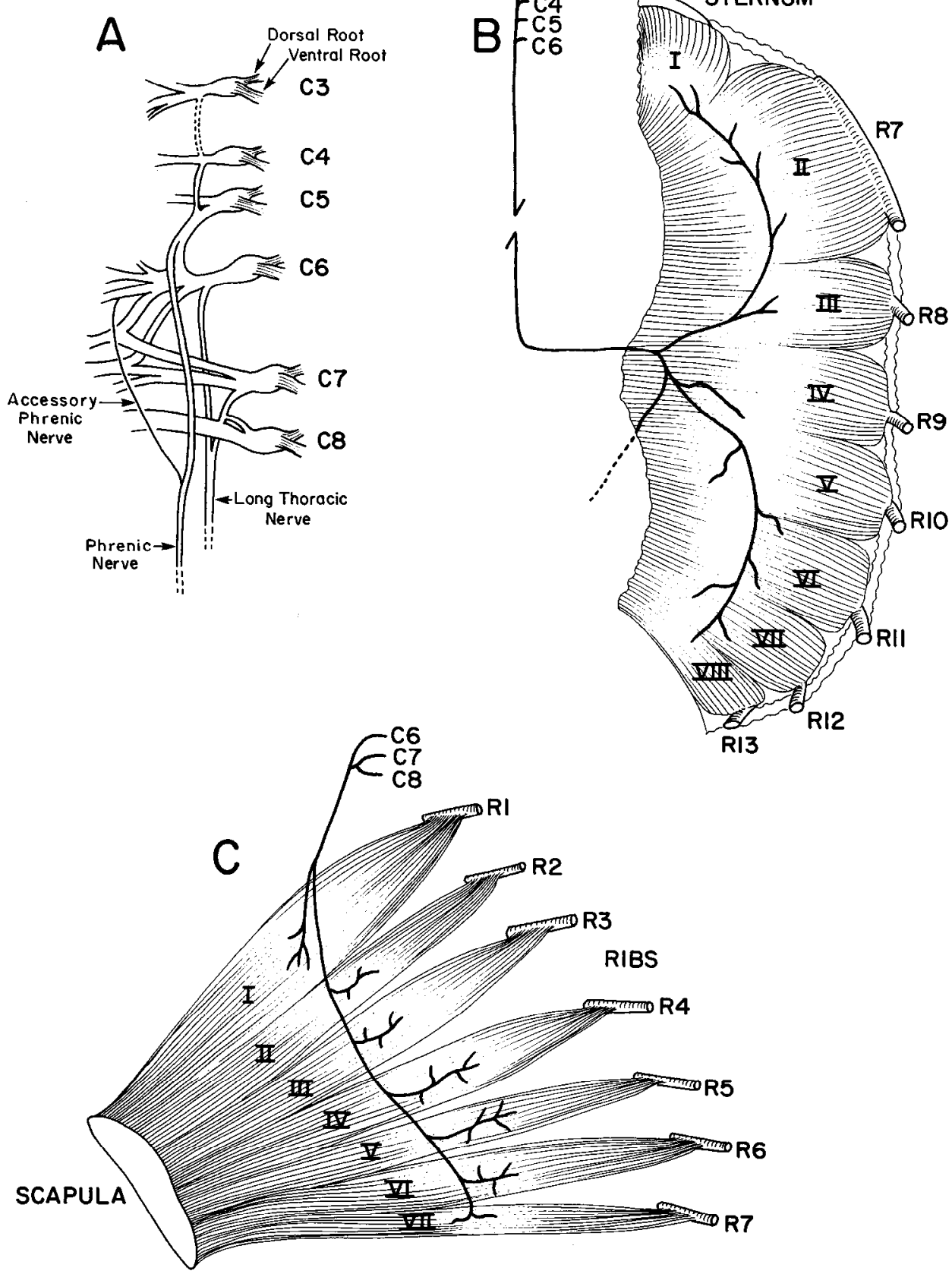

Figure 1. A, Contributions of cervical spinal nerves to the phrenic and the long thoracic nerves. The phrenic nerve is formed by branches from $\mathrm{C} 4-\mathrm{C} 6$; $\mathrm{C} 6$ projects to the phrenic through the accessory phrenic nerve. A branch from C3 (dotted) was found in 2 of 10 animals. The long thoracic is formed by branches from $\mathrm{C} 6-\mathrm{C} 8$. $B$, Diaphragm muscle, illustrating the 8 sectors formed by the origin of its fibers from connective tissue associated with the sternum and with ribs $7-13$. The phrenic nerve divides into rostral (upward) and caudal (downward) branches as it enters the diaphragm. A third major branch (dotted line) innervates the crus, which is anatomically distinct from the major portion of the diaphragm and was not studied. $C$, Anterior serratus muscle illustrating the seven sectors formed by the origin of its fibers from ribs 1-7. Each muscle segment receives a small branch from the long thoracic nerve. Drawings are not to scale.
For the diaphragm, $\mathrm{C} 4$ and $\mathrm{C} 5$ were each subdivided into rostral and caudal components. Ventral roots, or portions of roots, were pulled into suction electrodes for stimulation. The muscle was transilluminated and viewed through a dissecting microscope while square-wave pulses of 2 $10 \mathrm{~V}$ and $0.1 \mathrm{msec}$ duration were delivered through each suction elecrode in turn. The relative vigor of contractions evoked in each muscle slip (see Fig. 1, $B$ and $C$ ) by these stimuli was rated on a scale of $0-4$, with 4 being the strongest response in each muscle.

Following analysis of contractions, serratus muscles were paralyzed by exposure to $2 \mathrm{M}$ formamide for $20 \mathrm{~min}$ (Herrera, 1984) and then washed for $1 \mathrm{hr}$ before recording. Formamide is thought to paralyze muscle fibers by separating the transverse tubular network from the surface membrane (Agiro, 1981). The diaphragm was unexpectedly insensitive to formamide, requiring more than $1 \mathrm{hr}$ to block twitches and escaping from paralysis within $30 \mathrm{~min}$. Consequently, diaphragm muscles were stretched to prevent visible twitching of the muscle fibers. Muscle fibers were then impaled with a $3 \mathrm{M} \mathrm{KCl-filled} \mathrm{micropipette} \mathrm{for}$ intracellular recording. For each muscle fiber penetrated, ventral rootlets were stimulated individually and synaptic potentials recorded.

Immunocytochemistry. Immunocytochemical methods were used to lccate degenerated axons and denervated muscle fibers following section of individual ventral roots. An animal was injected with chloral hydrate and a dorsal laminectomy was performed. The dura mater was opened and the ventral root of either C4, C5, or C6 was cut. Damage to the dorsal root was inevitable with this approach but was limited to the region proximal to the dorsal root ganglion. Five days later, the animal was sacrificed and portions of the right phrenic nerve and hemidiaphragm were dissected. Tissues were frozen, sectioned in a cryostat, stained with antibodies, and viewed with epifluorescence illumination, all as detailed by Covault and Sanes (1986). Cross sections of nerve were doubly staincd with rabbit antibody to laminin (prepared using mouse laminin from Miles, Elkhart, IN, as immunogen) and mouse anitbody to neurofilaments (Glicksman and Willard, 1985), followed by fluorescein-conjugated goat anti-rabbit Ig and rhodamine-conjugated 


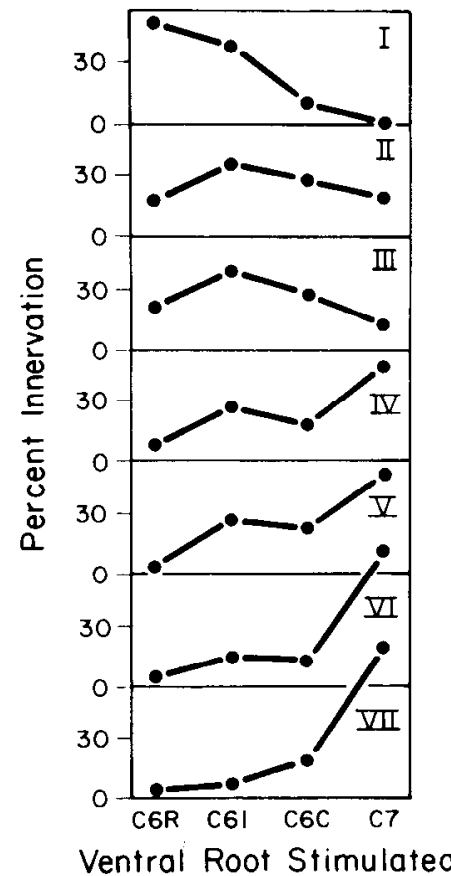

Figure 2. Segmental origin of synaptic inputs to the 7 sectors of the anterior serratus muscle. The figure represents the percentage of muscle fibers in each muscle sector (I-VII; see Fig. 1C) innervated through each ventral root or bundle of rootlets. Results from 10 muscles are combined; in general, synaptic potentials were recorded from 10 muscle fibers in each sector of each muscle.

goat anti-mouse $\operatorname{Ig}$ (Cappel, Cochranville, PA). Cross sections of muscle were stained with affinity-purified rabbit antibodies to rat N-CAM (Covault and Sanes, 1986), followed by fluorescein-conjugated goat antirabbit Ig and rhodamine- $\alpha$-bungarotoxin; N-CAM accumulates in denervated muscle fibers (Covault and Sanes, 1985). Only muscle sections devoid of bungarotoxin-stained endplates were analyzed, because N-CAM is present in synaptic regions of innervated as well as denervated muscle fibers (Covault and Sanes, 1986).

Retrograde labeling of motor neurons. Motor neurons innervating rostral and caudal parts of the diaphragm were labeled by retrograde transport of the fluorescent dyes, Nuclear yellow and Fast blue (Kuypers et al., 1980). Ten percent solutions of Nuclear yellow or Fast blue dye in $10 \%$ dimethylsulfoxide were injected into either the extreme rostral (muscle segment II in Fig. $1 B$ ) or caudal (muscle segments VI and VII) portions of the right hemidiaphragm. Injections were made in multiple, closely spaced sites on the abdominal surface of the muscle. The total amount of dye injected per diaphragm was 2-5 $\mu$ l. After a period of 3 $\mathrm{d}$, the rat was reanesthetized and perfused first with $0.9 \% \mathrm{NaCl}$, then with $4 \%$ paraformaldehyde in $0.1 \mathrm{~m}$ phosphate at $\mathrm{pH} 7.3$ for $10 \mathrm{~min}$. The cervical spinal cord was removed from $C 2$ to $C 7$, fixed in $4 \%$ paraformaldchydc for an additional $30 \mathrm{~min}$, rinsed, embedded in gelatin, and sectioned on a Vibratome at $50 \mu \mathrm{m}$ thickness. Sections were mounted on slides and examined with epifluorescence optics. The 2 dyes were easily distinguished by color and subcellular location (i.e., yellow nuclei vs blue cytoplasm).

\section{Results}

\section{Topographical innervation of the serratus anterior}

The aim of this study was to ask whether and how the rostrocaudal axis of a spinal motor pool is mapped onto the rostrocaudal axis of a skeletal muscle. For this purpose, we sought muscles that are supplied by a single peripheral nerve and in which the relative rostrocaudal positions of both motor neurons and muscle fibers are easy to define. The serratus anterior fulfills these criteria. Fibers in this muscle originate from the medial anterior portions of the first 7 ribs and are thereby divided into

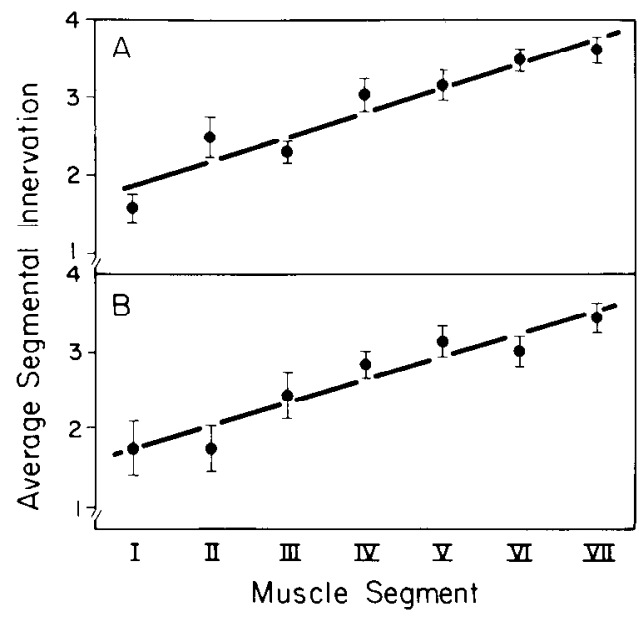

Figure 3. Average segmental innervation of the 7 sectors of the anterior serratus. A, Average segmental innervation of each muscle sector calculated from synaptic inputs recorded intracellularly, as described in Results. Points and bars represent the mean value obtained from 10 muscles, $\pm 1 \mathrm{SE}$ of the mean. $B$, Similar index, calculated from contractile responses evoked by stimulation of ventral roots or rootlets, as described in Matcrials and Methods. The solid lines are calculated slopes of data points: 0.32 for $A$ and 0.30 for $B$, both of which are different from zero at the $p<0.01$ level.

7 slips, each of which has a distinct rostrocaudal position relative to the others (Fig. 1C). The muscle is innervated through a single nerve, the long thoracic, which in turn derives from roots $\mathrm{C} 6$, $\mathrm{C} 7$, and $\mathrm{C} 8$, each of which can be stimulated individually (Fig. 1A). Preliminary experiments revealed that C6 contributes the bulk of the innervation to the serratus, but we found that it was possible to subdivide this root reproducibly, along natural divisions, into rostral $(R)$, intermediate $(I)$, and caudal $(C)$ bundles of rootlets. We therefore stimulated the 3 divisions of $\mathrm{C} 6$, as well as $\mathrm{C} 7$ and $\mathrm{C} 8$, and recorded synaptic inputs from fibers in each of the 7 divisions of the serratus anterior.

Results of this analysis are summarized in Figure 2. It is apparent that muscle fibers in any sector can receive synaptic input from any part of the motor pool. However, the rostral and caudal portions of the motor pool preferentially innervated the rostral and caudal portions, respectively, of the serratus anterior muscle. For example, muscle segment $I$, the most rostral, received $50 \%$ of its innervation from C6R and only $1 \%$ from $\mathrm{C} 7$. The most caudal muscle sector (VII), on the other hand, received only $3 \%$ of its innervation from C6R, but $73 \%$ from $\mathrm{C7}$. Intermediate muscle sectors were innervated by intermediate subsets of axons. (No synaptic potentials were evoked by stimulation of $\mathrm{C} 8$; presumably this root contributes only sensory fibers to the long thoracic ncrve.)

To ask whether the motor pool-to-muscle map was relatively continuous, or whether it harbored major discontinuities, we constructed an index that reflects the average segmental origin of all synaptic inputs to each division of the serratus anterior. Stimulated groups of axons were numbered in rostrocaudal or$\operatorname{der}(\mathrm{C} 6 \mathrm{R}=1, \mathrm{C} 6 \mathrm{I}=2, \mathrm{C} 6 \mathrm{C}=3, \mathrm{C} 7=4)$, and these numbers used to calculate a positionally weighted average of the inputs we recorded. For example, a hypothetical sector in which 5 muscle fibers are innervated by $\mathrm{C} 6 \mathrm{I}$ and 5 by $\mathrm{C} 7$ would receive a value of $[5(2)+5(4)] / 10=3.0$. In this index, muscle sectors innervated by more caudally derived subsets of axons receive higher values of segmental innervation. Use of this index re- 


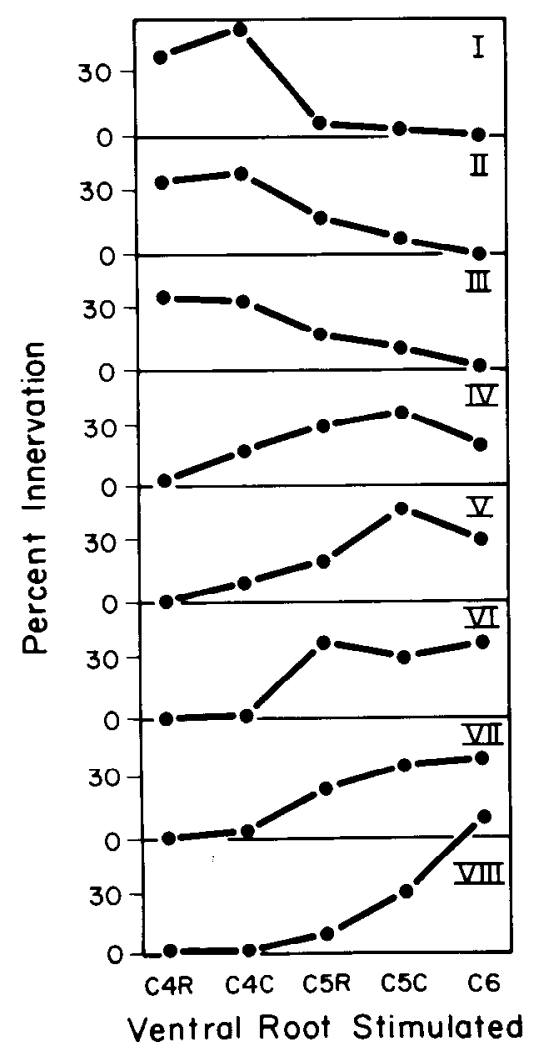

Figure 4. Segmental origin of synaptic inputs to the 8 sectors of the diaphragm. The figure represents the percentage of muscle fibers in each sector (I-VIII; see Fig. 1B) innervated through each ventral root or bundle of rootlets. Synaptic potentials were recorded from 10 muscle fibers in each sector of 10 muscles.

vealed a regular progression in the average segmental innervation of successive muscle sectors (Fig. $3 A$ ). Similar results were obtained using an index derived from visual estimates of muscle contractions evoked by stimulation of individual ventral roots or rootlets (Fig. $3 B$ ). Thus, the rostrocaudal axis of the serratus motor pool is mapped continuously onto the rostrocaudal axis of the serratus muscle.

\section{Topographical innervation of the diaphragm}

To ask whether rostrocaudal maps of motor pools onto muscles occur elsewhere in the body, we studied the segmental innervation of the diaphragm. While this muscle is obviously oriented in the dorsal-ventral axis, its fibers in fact originate from connective tissue associated with the sternum and with ribs 7-13; it is thereby divided into 8 sectors, which form a clear rostrocaudal progression (Fig. 1B). Furthermore, it is innervated through a single nerve, the phrenic, which receives contributions from several spinal segments (Fig. 1A). Finally, electromyographic studies in cats and dogs had already indicated that rostral and caudal roots preferentially innervated rostral and caudal portions, respectively, of the diaphragm (Duron et al., 1979; Landau et al., 1982). We therefore divided the major roots into rostral ( $\mathrm{R}$ ) and caudal $(\mathrm{C})$ parts along natural subdivisions, stimulated individual roots or rootlets $(\mathrm{C} 3, \mathrm{C} 4 \mathrm{R}, \mathrm{C} 4 \mathrm{C}, \mathrm{C} 5 \mathrm{R}, \mathrm{C} 5 \mathrm{C}$, and $(6)$, and recorded synaptic potentials from muscle fibers in each of the 8 sectors of the diaphragm.

Our results, computed in Figures 4 and 5, revealed that the rostrocaudal axis of the phrenic motor pool is systematically

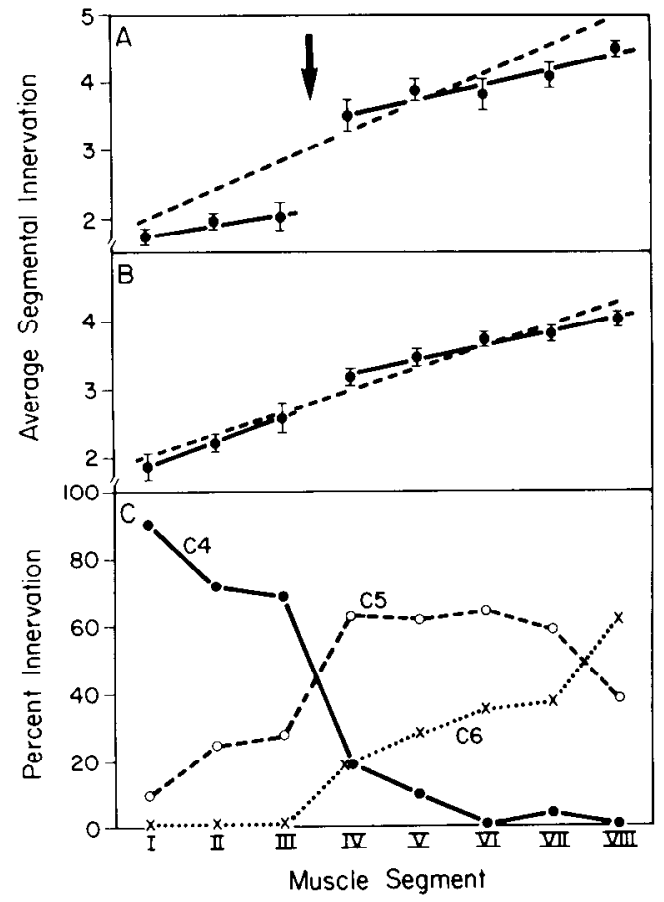

Figure 5. $A$ and $B$, Average segmental innervation of the 8 sectors of the diaphragm calculated as in Figure 3 using synaptic inputs recorded intracellularly $(A)$ or visual estimates of contractile vigor $(B)$. The large arrow marks the position where the phrenic nerve bifurcates into rostral and caudal branches (see Fig. $1 B$ ). Lines were calculated by linear regression. In $A$, the dashed line is the calculated slope $(0.428$, different from 0 at $p<0.01$ by Student's $t$ test) for all data points, indicated by solid dots \pm 1 SEM. The solid line through segments I-III and IV-VIII also have slopes $(0.150$ and 0.222$)$ significantly different from zero $(p<$ $0.05)$. In $B$, the calculated slopes for all muscle segments $(0.324)$ for rostral segments $(0.360)$ and caudal segments $(0.207)$ were all significantly different from zero at the $p<0.01$ level. $C$, Percentage innervation of each muscle segment by $\mathrm{C} 4, \mathrm{C} 5$, and $\mathrm{C} 6$ (calculated from data in Fig. 4).

mapped onto the rostrocaudal axis of the diaphragm. Thus, muscle sector I received $90 \%$ of its innervation from $\mathrm{C} 4$ and virtually none from $\mathrm{C} 6$; sector IV received most $(63 \%)$ of its innervation from $\mathrm{C} 5$; and sector VIII received most (62\%) of its innervation from C6. C3 detectably innervated only 2 of the 10 muscles we studied, and is therefore omitted from Figure 4, but in these 2 muscles, it innervated rostral muscle sectors exclusively. Thus, the diaphragm, like the serratus anterior, is topographically innervated.

Construction of an index of segmental innervation for the diaphragm (Fig. 5A) confirmed the systematic nature of the motor pool-to-muscle projection but additionally revealed a major discontinuity in the map, between sectors III and IV: Muscle fibers in sector IV are innervated by a considerably more caudally derived subset of axons than are fibers in sector III, while differences within rostral (I-III) or caudal (IV-VIII) groups of sectors are small. This discontinuity was also evident, though less pronounced, in the index calculated from visual estimates of contractile vigor (Fig. $5 B$ ). Analysis of the contribution of each ventral root to the innervation of each muscle sector indicates that all 3 roots contribute to the discontinuity (Fig. 5C). An obvious anatomical correlate of the discontinuity is the bifurcation of the phrenic nerve into rostroventrally and caudodorsally directed branches, which occurs near the border between sectors III and IV (Fig. $1 B$ ). This correlation suggests that 

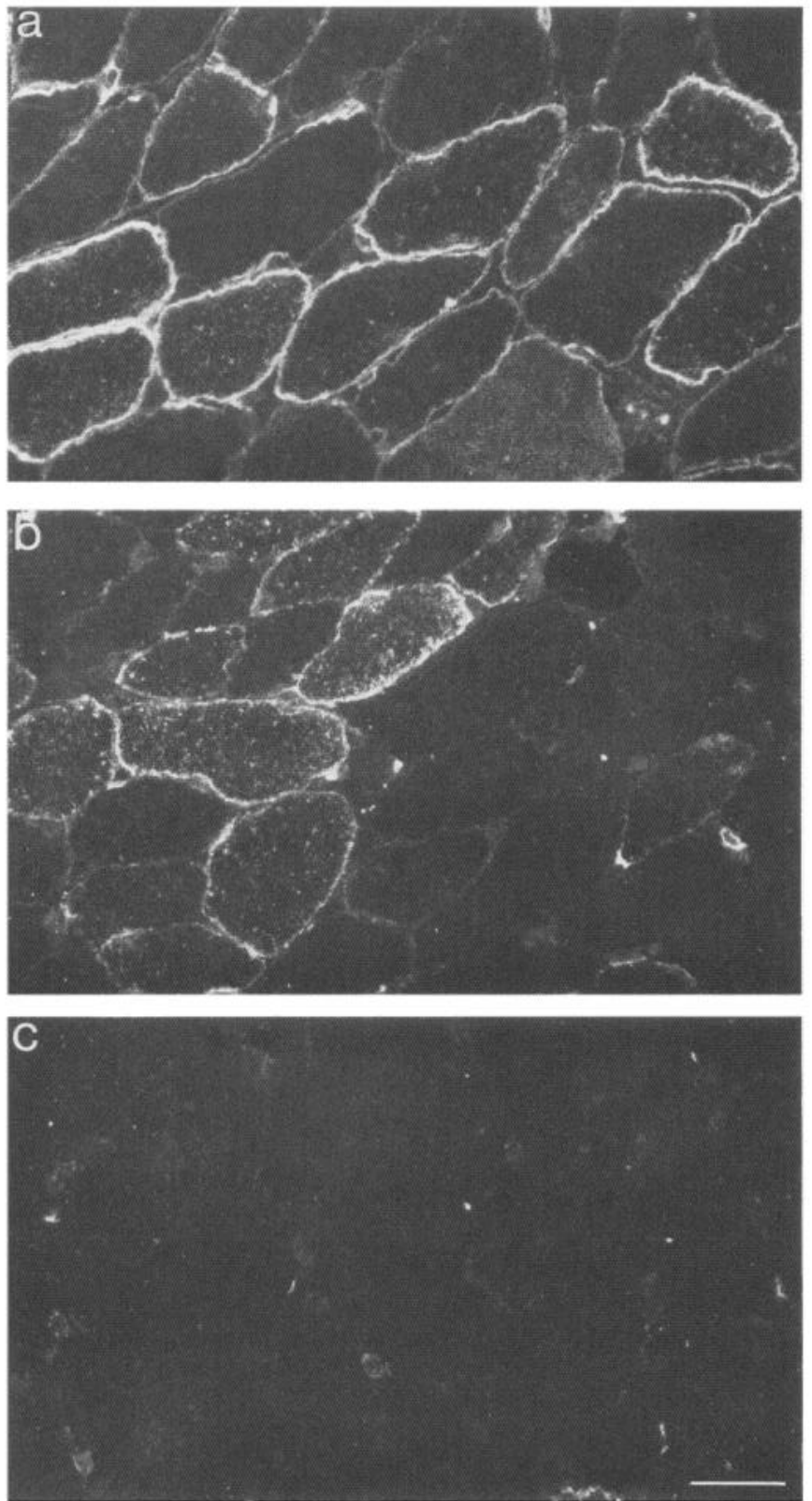

Figure 6. Fluorescent-labeled anti-N-CAM in a partially denervated diaphragm, $5 \mathrm{~d}$ after transection of the $\mathrm{C} 4$ ventral roots. Sections taken from rostral (sector I), intermediate (sector III), and caudal (sector V) portions of the diaphragm (corresponding to Fig. 6, $A-C$, respectively) were stained with anti-N-CAM and second antibody. Anti-N-CAM binding, which increases in denervated muscle fibers, was greatest in rostral muscle fibers. Note discrete border in sector III $(B)$, between areas of predominantly denervated and predominantly innervated muscle fibers. A second experiment produced similar results.

axons from different segments may exhibit markedly different directional preferences at the branch point, a possibility we address directly below. It is important to note, however, that the overall topography of the diaphragm's innervation (dotted lines in Fig. 5, $A, B$ ) cannot be accounted for by the discontinuity at the branch point: There is a significant topographical projection within both rostral (I-III) and caudal (IV-VIII) groups of sectors (solid lines in Fig. 5, $A, B$, and see Fig. 5 legend). Thus, both a graded component distributed throughout the muscle and a discontinuous component near the nerve's bifurcation

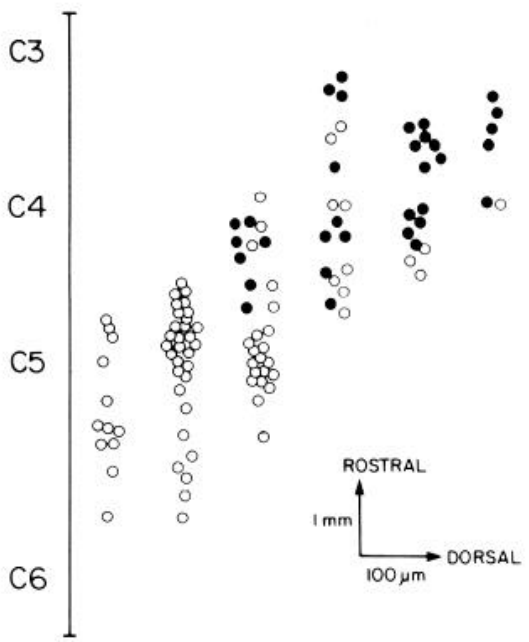

Figure 7. Position of fluorescent dye-labeled motoneuron cell bodies in the phrenic motor nucleus following injection of Fast blue into sectors VI and VII of the diaphragm and Nuclear yellow into the rostral portion of sector II. Each column represents a single longitudinal section of the cervical cord. Sections are progressively more dorsal from left to right. Fast blue-filled motoneurons (open circles) are concentrated in caudal portions of the cord, while Nuclear yellow-filled cell bodies (closed circles) are localized to rostral portions of the spinal cord. Figure represents data from 1 experiment $3 \mathrm{~d}$ after dye injection. Similar results were obtained in a second experiment.

contribute to the projection of the rostrocaudal axis of the phrenic motor pool onto the rostrocaudal axis of the diaphragm.

\section{Histological assays of topographical innervation}

We used 2 histological methods to extend our electrophysiological analysis of the diaphragm. In the first method, single ventral roots were cut and the consequent partial denervation was mapped by exploiting the finding that levels of N-CAM increase in denervated muscle fibers (Covault and Sanes, 1985). Either C4 or C6 ventral roots were transected; $5 \mathrm{~d}$ later, the right hemidiaphragms were sectioned and stained with an antibody to muscle N-CAM. As shown in Figure 6, transection of the ventral root from $\mathrm{C} 4$ resulted in a selective increase in immunohistochemically detectable N-CAM in rostral portions of the diaphragm. Conversely, section of $\mathrm{C} 6$ produced selective denervation of caudal muscle sectors (not shown). In addition, anti-N-CAM staining following section of $\mathrm{C} 4$ revealed a sharp boundary between predominantly innervated and predominantly denervated zones in sectors III (Fig. $6 \mathrm{~B}$ ) or IV; this boundary is likely to correspond to the discontinuity detected electrophysiologically and described above. Thus, immunocytochemical analysis independently confirmed our electrophysiological results.

In a second histological study, the phrenic motor pool in the spinal cord was labeled retrogradely following injection of dyes into the diaphragm. Our purpose here was to exclude the possibility that motoneurons are randomly arranged in the motor column but that their axons are sorted intraspinally before exiting through the ventral roots. There is, in fact, considerable evidence that motor (and autonomic preganglionic) axons emerge from the spinal cord close to the level of their cell bodies (e.g., Sherrington, 1892; Sprague, 1948; Rubin and Purves, 1980; Smith and Hollyday, 1983), but we considered it important to test this assumption for the phrenic motor nucleus. We therefore injected Fast blue into the caudal region and Nuclear yellow 

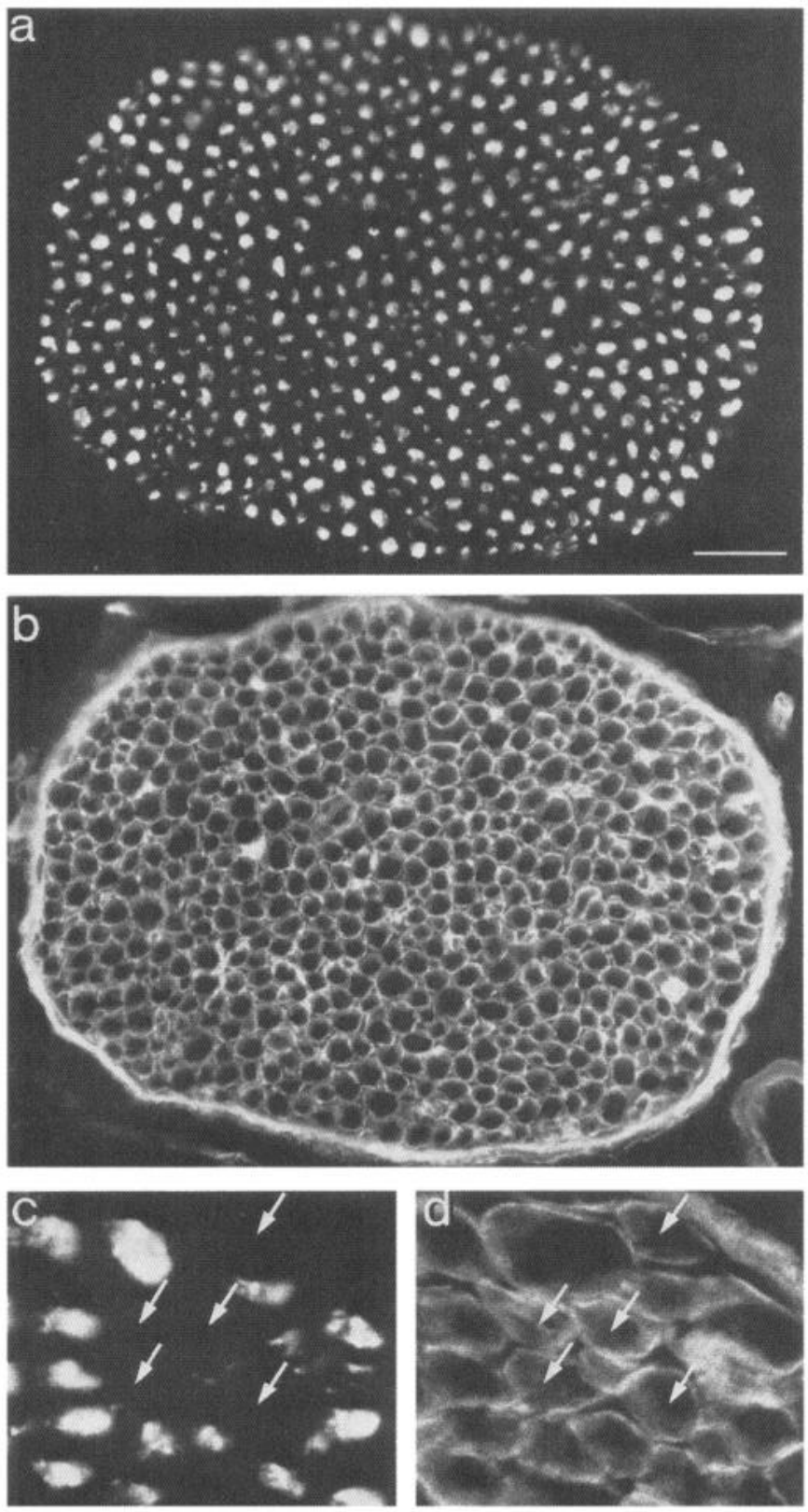

Figure 8. Immunocytochemical identification of degenerated axons in phrenic nerve. Sections incubated with rabbit anti-laminin plus mouse anti-neurofilaments, then with fluorescein-anti-rabbit Ig plus rhodamine-anti-mouse Ig, were photographed with rhodamine $(a$ and $c$ ) or fluorescein $(b$ and $d)$ optics. $a$ and $b$, A section of an intact phrenic nerve. $c$ and $d$, An enlarged area of the phrenic nerve from an animal that had the ipsilateral C5 ventral root sectioned $5 \mathrm{~d}$ earlier. Arrows indicate that some basal lamina sheaths $(d)$ no longer contain neurofilaments $(c)$ and are thus identifiable as the endomysia of degenerated axons. Scale bars, $25 \mu \mathrm{m}$ for $a$ and $b ; 10 \mu \mathrm{m}$ for $c$ and $d$.

into the rostral region of the same hemidiaphragm, waited $3 \mathrm{~d}$ for retrograde transport, and then mapped labeled motoneurons in the cervical spinal cord. A reconstruction of the phrenic motor column from 1 such animal is illustrated in Figure 7. The extent of the column, from $\mathrm{C} 3$ to $\mathrm{C} 6$, and its slight rostrodorsal to caudoventral slant are in agreement with a report by Gottschall (1981). Fast blue-labeled motoneurons were localized in the more caudal region of the motor pool, while Nuclear yellowlabeled cells were localized in more rostral regions of the column.

\begin{tabular}{|c|c|c|c|c|}
\hline \multirow{2}{*}{$\begin{array}{l}\text { Sec- } \\
\text { tioned } \\
\text { ven- } \\
\text { tral } \\
\text { root }\end{array}$} & \multirow[b]{2}{*}{ Exp. } & \multirow{2}{*}{$\begin{array}{l}\text { Total } \\
\text { large } \\
\text { axons } \\
(n)\end{array}$} & $\begin{array}{l}\text { Degenerated } \\
\text { Axons }\end{array}$ & \multirow{2}{*}{$\begin{array}{l}\text { Mean } \\
\text { per- } \\
\text { cent }\end{array}$} \\
\hline & & & $n \quad(\%)$ & \\
\hline \multirow[t]{3}{*}{$\mathrm{C} 4$} & 1 & 454 & $95(21)$ & \\
\hline & 2 & 382 & $146(38)$ & \\
\hline & 3 & 480 & $121(25)$ & 28 \\
\hline C5 & 4 & 373 & $146(39)$ & 39 \\
\hline \multirow[t]{2}{*}{$\mathrm{C} 6$} & 5 & 391 & $13(3)$ & \\
\hline & 6 & 389 & $14(4)$ & 3 \\
\hline \multicolumn{3}{|c|}{ Total degenerated axons } & & 70 \\
\hline
\end{tabular}

Thus, the 50 most caudal fluorescent motoneurons were all blue, while 28 of the 37 most rostral labeled cells were yellow. Blue and yellow cells overlapped in the middle of the motor column, especially in the area between the $\mathrm{C} 4$ and $\mathrm{C} 5$ ventral roots. We do not know whether this overlap represents a real mixing of motoneurons (which would be consistent with the electrophysiological results) or is due to diffusion of dye within the diaphragm. In either case, however, this histological result confirms that the rostrocaudal projections demonstrated by stimulation or section of ventral roots reflect a projection of rostrally and caudally situated motoneuronal somata to rostral and caudal portions, respectively, of the muscle.

\section{Arrangement of motor axons in the phrenic nerve}

The existence of orderly projections of motor pools onto muscles raises at least 2 questions about the arrangement of motor axons within peripheral nerves. First, in that both motor neurons and their axon terminals form corresponding arrays, we wondered whether order is maintained along the length of the nerve or whether axons disperse within the nerve and then reorganize near or within the muscle. Second, in that the projection to the diaphragm displayed a major discontinuity near the point of nerve entry (Fig. 5), we wondered whether axons from different roots might make different "choices" at the bifurcation of the nerve. To address these questions, we devised a method to follow the course of axons from a single root within the phrenic nerve. A single ventral root was severed, and after $5 \mathrm{~d}$ the animals were sacrificed and specific regions of the phrenic nerve were sectioned and double-stained with antibodies to laminin (a component of the Schwann cell basal lamina) and to neurofilaments (a component of axoplasm). In normal nerves, large myelinated axons such as motor axons are rich in neurofilaments and each axon is encircled by a Schwann cell, which is in turn ensheathed by basal lamina (Fig. 8, $a, b$ ). After axotomy, the basal lamina sheath survives, while the axon degenerates (Thomas, 1964). Thus, following partial denervation, basal lamina sheaths that contain no neurofilament-positive structures mark the sites of degenerated axons (Fig. 8, $c, d$ ). Table 1 lists the numbers of degenerating axons ("empty" basal lamina sheaths) and the total number of large axons (total basal lamina sheaths) in the phrenic nerve of each of 6 animals studied after section of a single ventral root. An average of $28 \%$ of the axons degenerated following section of $\mathrm{C} 4,39 \%$ following section of $\mathrm{C} 5$, and $3 \%$ following section of $\mathrm{C} 6$. The total percentage of large fibers affected by ventral root section was $70 \%$. This num- 

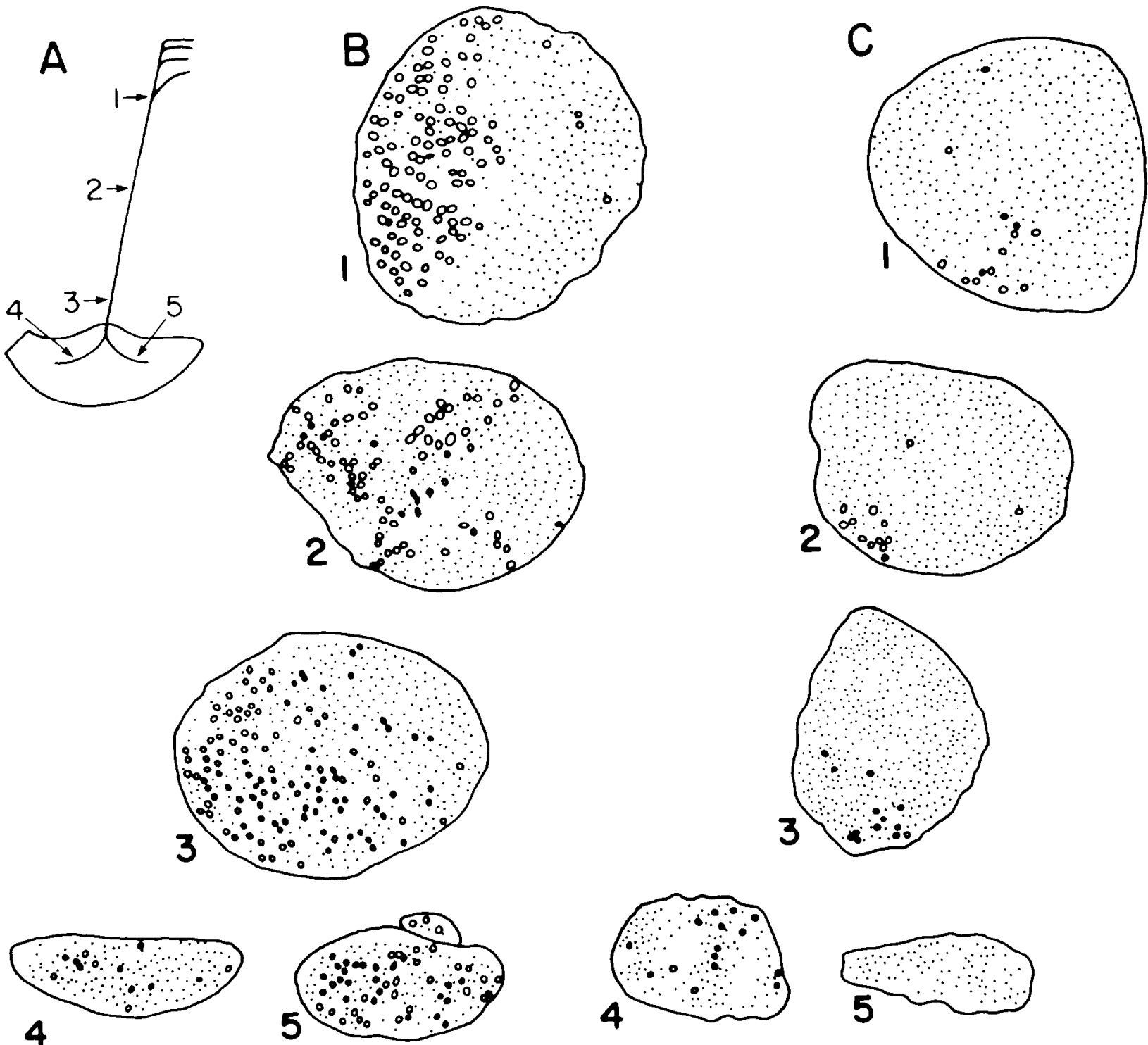

Figure 9. Location of axons from $\mathrm{C} 4(B)$ and $\mathrm{C} 6(C)$ within the phrenic nerve, determined immunohistochemically following section of individual ventral roots. $A$, Five regions of the phrenic nerve from which sections were taken: ( 1$)$ just distal to the entry of the accessory phrenic, (2) at the level of entry of the venae cavae into the right atrium, (3) just proximal to the bifurcation of the phrenic nerve, (4) caudal intramuscular branch, and (5) rostral intramuscular branch. $B$ and $C$, Tracings from micrographs of doubly stained sections such as those shown in Figure 8 . In each section small dots indicate axons represented by laminin-ensheathed neurofilament-positive structures. Circles indicate basal lamina sheaths that did not have corresponding neurofilament reactivity and thus represent degenerated fibers. A consistent finding among all $\mathrm{C}_{4}$ and $\mathrm{C}_{6}$ sectioned animals was that axons from a single ventral root tend to cluster in extramuscular but not intramuscular portions of the nerve. Degenerating axons were more numerous in the rostral $(48 \%)$ than in the caudal $(12 \%)$ intramuscular branch following section of root C4 in 2 experiments. In 1 experiment, degenerating axons were found in the caudal intramuscular branch (12\%) but not in the rostral branch following section of root C6. (Sections from positions 1-3 are arbitrarily oriented with degenerated axons to the bottom and left; the true orientation of these sections was lost during processing.)

ber agrees with Gottschall's (1981) estimate that $71 \%$ of the large axons in the phrenic nerve are motor axons and supports the idea that our immunocytochemical method selectively and reliably identifies degenerating motor axons in the phrenic nerve.

Figure 9 illustrates double-labeled sections from several levels of the phrenic nerve following section at ventral roots $\mathrm{C} 4$ or $\mathrm{C} 6$. Three conclusions can be drawn from these (and 4 other) reconstructions. First, in each case axons from a single root are loosely clustered within the nerve. Some degree of clustering is maintained along the length of the extramuscular portion of the nerve (compare sections 1, 2, and 3 in Fig. 9, $B$ and $C$ ). Second, the compartmentalization of degenerating axons is not strict. In both animals in which $\mathrm{C} 6$ was sectioned 1 or 2 degenerating axons were located far from the cluster, and numerous intact axons were intermixed with degenerating axons in all cases. In addition, neither $\mathrm{C} 4$ nor $\mathrm{C} 6$ axons were obviously clustered within intramuscular branches (Fig. 9, sections 4 and 5). Finally, axons from different ventral roots take markedly different courses when the nerve enters the muscle and bifurcates. Four times more degencrating axons were found in the rostral intramuscular branch than in the caudal branch following damage to $\mathrm{C4}$; no degenerating axons were found in the rostral branch, but several in the caudal branch, following damage to $\mathrm{C} 6$. Thus, the nonrandom distribution of motor axons within the phrenic nerve 
and the divergent projections of axons at the nerve's bifurcation may both contribute to the orderly synaptic topography within the muscle.

\section{Discussion}

The neuromuscular system has obvious technical advantages for studies of how patterns of connectivity between neurons and their targets are generated and maintained. Studies of the innervation of the limb have revealed great specificity and considerable topographic order in the matching of spinal motor pools to limb muscles (e.g., Sherrington, 1892; Browne, 1950; Hollyday, 1980; Weeks and English, 1985), and studies of embryonic limbs have provided evidence for the existence of cues that guide axons en route to and possibly within muscles (reviewed in Landmesser, 1984). Our aim was to explore a potentially simpler pattern, the projection of a single motor pool onto a single muscle. Previous studies have shown that a number of limb muscles receive topographically organized projections from their motor pools (e.g., Swett et al., 1970; Brown and Booth, 1983; Bennett and Lavidis, 1984). Here, we have identified and studied 2 muscles, the diaphragm and serratus anterior, that we believe are particularly well suited for investigations of such synaptic patterns: They are trunk, rather than limb, muscles; they are thin and flat (i.e., essentially 2 -dimensional); they are divided into a series of sectors that form a rostrocaudal progression; and each is supplied by a single nerve. We found that for both muscles the rostrocaudal axis of the motor pool is systematically mapped onto the rostrocaudal axis of the muscle. In addition, for one muscle, the diaphragm, we studied the segmental arrangement of motor axons within extra- and intramuscular portions of its nerve. The synaptic and axonal arrangments we document, together with previous results (Wigston and Sanes, 1982, 1985), suggest several factors that may contribute to the motor pool-to-muscle map.

Phrenic motor axons that enter the phrenic nerve through any one ventral root remain loosely clustered along the length of the nerve until they enter the diaphragm. Axons from individual segmental levels have previously been shown to remain clustered within large peripheral nerve trunks in dogs (Ueyama, 1978) and chickens (Stirling and Summerbell, 1979; Lance-Jones and Landmesser, 1981); our results show that a similar compartmentalization is detectable within a nerve that innervates a singlc muscle. In the optic nerve of lower vertebrates, where considerable fiber order has also been demonstrated, axonal topography has been postulated to arise from temporal patterns of axon growth and to contribute to the formation of the retinotectal map (e.g., Reh et al., 1983, and references therein). We have no information on when and how motor axons from different segmental levels enter the phrenic nerve, and we do not know whether the grouping of axons is a consequence of spatiotemporal patterns of growth or reflects interactions among growing axons (or their ensheathing cells). In either case, it is possible that segmentally selective fasciculation could play a role in guiding later arising axons to appropriate parts of the nerve or muscle or in amplifying axonal responsiveness to weak extrinsic cues that single axons may be unable to detect reliably.

The phrenic nerve bifurcates as it enters the diaphragm. C4 motor axons preferentially enter the rostrally directed branch, while virtually all C6 axons enter the caudally directed branch. The binary choice that axons make at this site is likely to account for the discontinuity in the projection of the phrenic motor pool onto the diaphragm (Fig. $5 A$ ) and to underlie, in part, the matching of rostral and caudal portions of the motor pool with rostral and caudal portions, respectively, of the muscle. Similarly, choices that limb motorneurons make within the crural and ischiadic plexuses contribute importantly to the specificity of innervation of limb muscles (Lance-Jones and Landmesser, 1981). In this case, axons are actively guided through the plexus; that is, their routes cannot be accounted for by temporal or spatial patterns of growth (Lance-Jones and Landmesser, 1981). It remains unknown whether specific interactions must likewise be postulated to account for the divergent choices that $\mathrm{C} 4$ and C6 axons make at the bifurcation of the phrenic nerve. In any event, however, the simple geomctry of the phrenic ncrve at its bifurcation into rostral and caudal branches will make it a useful site at which to search for the structures with which developing axons interact.

Within the serratus anterior and within each quadrant of the diaphragm, progressively more caudal muscle sectors are innervated by progressively more caudally derived subsets of motor axons. This mapping of the motor pool onto the muscle surface clearly does not involve rigidly specified connectivity, in that axons from virtually any part of the motor pool can innervate fibers in any part of the muscle. Instead, the specificity is statistical in nature, with axons from a particular portion of the motor pool being most likely to innervate a corresponding portion of the muscle, and gradually less likely to innervate more distant portions. A similar orderly but nonrigid specificity has been documented by Purves and colleagues in the projection of autonomic prcganglionic axons from different segmental levels to neurons within single sympathetic ganglia (e.g., Njå and Purves, 1977). In muscle, such a map might arise in any of several ways, including selective recognition of neuron and muscle fibers, intramuscular guidance of axons to appropriate points of termination, or coordinated spatiotemporal patterns of nerve and muscle growth; all 3 alternatives have been considered in studies of the retinotectal map (see Cowan and Hunt, 1985, for review). However, 3 previous results argue for the existence of positional labels in or near muscle fibers. First, we have shown that intercostal muscles transplanted from different segmental levels to a common site can be preferentially reinnervated by autonomic preganglionic axons from corresponding segmental levels of the spinal cord (Wigston and Sanes, 1982, 1985). Second, Bennett and Lavidis (1984) and Brown and Booth (1983) have reported that elimination of polyneuronal innervation from neonatal limb muscles is segmentally selective. Third, Wigston (1986) has recently demonstrated segmentally selective reinnervation of transplanted axolotl limb muscles by motor axons. Together, these results argue that at least postnatally, some structures within skeletal muscles bear labels that impart a segmental bias to synapse formation or maintenance. Our present results provide no insight into where these labels might be: Possibilities include muscle fibers, extracellular matrix, and intramuscular nerve branches. A hypothesis consistent with our previous results (Wigston and Sanes, 1982, 1985) is that motor neurons and muscle fibers vary in some positional factor that promotes connectivity among synaptic partners from corresponding levels in the rostrocaudal axis.

In conclusion, our results provide 3 candidates that may help to explain the mapping of motor pools onto muscles: segmental clustering within the peripheral nerve, guided choices of axons at nerve branch points, and positional labels within muscles. Larger tasks will be to learn whether and to what extent these factors contribute to the generation of the map during development, and whether any of them are active during reinnervation in the adult. Interestingly, these factors are similar to 
those that have already been invoked to explain 2 of the best studicd neural projections: the retinotopic map and the projection of groups of motor pools onto groups of muscles in the limb. Thus, fibers within the optic nerve display considerable order in their arrangement; axons are rearranged at branch points (e.g., the chiasm) in ways that suggest active guidance; and positional labels on the tectal surface probably guide axonal growth or bias synapse formation within the target area (e.g., Reh et al., 1983; Bonhoeffer and Gurer, 1984; Cowan and Hunt, 1985). Similarly, in the limb, axonal ordering, axonal guidance within the plexus, and local labels within the limb may all contribute to the specificity of innervation observed (e.g., Lance-Jones and Landmesser, 1981; Whitelaw and Hollyday, 1983; Landmesser, 1984). It is therefore possible that studies of the development of synaptic topography in single, flat muscles such as the diaphragm and serratus anterior will be useful in explaining the genesis of other neuronal maps.

\section{References}

Agiro, V. (1981) Excitation-contraction uncoupling of striated muscle fibers by formamide treatment: Evidence of detubulation. J. Muscle Res. Cell Motil. 2: 283-294.

Bennett, M. R., and N. A. I avidis (1984) Development of the topographical projection of motor neurons to a rat muscle accompanies loss of polyneuronal innervation. J. Neurosci. 4: 2204-2212.

Bonhoeffer, F., and A. Gurer (1984) How do retinal axons find their targets on the tectum? Trends Neurosci. 7: 378-381.

Brown, M. C., and C. M. Booth (1983) Postnatal development of the adult pattern of motor axon distribution in rat muscle. Nature 304 . 741-742.

Browne, K. M. (1950) The spatial distribution of segmental nerves to striate musculature of the hindlimb of the rat. J. Comp. Neurol. 93 . 441-455.

Covault, J., and J. R. Sanes (1985) Neural cell adhesion molecule (NCAM) accumulates in denervated and paralyzed skeletal muscles. Proc. Natl. Acad. Sci. USA 82: 4544-4548.

Covault, J., and J. R. Sanes (1986) Distribution of N-CAM in synaptic and extrasynaptic portions of developing and adult skeletal muscles. J. Cell Biol. 102: 716-730.

Cowan, W. M., and M. R. K. Hunt (1985) Development of retinotectal projections-an overview. In Molecular Bases of Neural Development, G. M. Edelman, W. E. Gall, and W. M. Cowan, eds., pp. 389-428, Wiley, New York.

Duron, B., D. Marlot, and J. M. Macron (1979) Segmental motor innervation of the rat diaphragm. Neurosci. Lett. 15: 93-96.

Glicksman, M. A., and M. Willard (1985) Differential expression of three neurofilament polypeptides. Ann. NY. Acad. Sci. 455: 479-491.

Gottschall, J. (1981) The diaphragm of the rat and its innervation. Muscle fiber composition; perikarya and axons of efferent and afferent neurons. Anat. Embryol. 161: 405-417.

Gottschall, J., and H. Gruber (1977) The accessory phrenic nerve in the rat. Anat. Embryol. 151: 63-69.

Herrera, A. A. (1984) Polyneuronal innervation and quantal transmitter release in formamide-treated frog sartorius muscles. J. Physiol. (Lond.) 355: 267-280.

Hollyday, M. (1980) Organization of motor pools in the chick lumbar lateral motor column. J. Comp. Neurol. 194: 143-170.
Kuypers, H. G. J. M., M. Bentivoglio, C. E. Catsman-Berrevoets, and A. T. Bharas (1980) Double retrograde neuronal labeling through divergent axon collaterals, using two fluorescent tracers with the same excitation wavelength which label different features of the cell. Exp. Brain Res. 40: 383-392.

Lance-Jones, C., and L. Landmesser (1981) Pathway selection by chick lumbrosacral motoneurons during normal development. Proc. R. Soc. Lond. [Biol.] 214: 1-18.

Landau, B. R., K. Akert, and T. S. Roberts (1962) Studies on the innervation of the diaphragm. J. Comp. Neurol. 119: 1-10.

Landmesser, L. (1984) The development of specific motor pathways in the chick embryo. Trends Neurosci. 7: 336-339.

Laskowski, N. B., and J. R. Sanes (1985) Topographic mapping of motor pools onto muscles. Soc. Neurosci. Abstr. 15:63.

Lichtman, J. W., D. Purves, and J. W. Yip (1979) On the purpose of selective innervation of guinea-pig supcrior cervical ganglion cells. J. Physiol. (Lond.) 292: 69-84.

Njå, A., and D. Purves (1977) Reinnervation of guinea pig superior cervical ganglion cells by preganglionic fibers arising from different levels of the spinal cord. J. Physiol. (Lond.) 272: 633-651.

Reh, T. A., E. Pitts, and M. Constantine-Paton (1983) The organization of the fibers in the optic nerve of normal and tectum-less Rana pipiens. J. Comp. Neurol. 218: 282-296.

Rubin, E., and D. Purves (1980) Segmental organization of sympathetic preganglionic neurons in the mammalian spinal cord. J. Comp. Neurol. 192: 163-174.

Sherrington, C. S. (1892) Notes on the arrangement of some motor fibers in the lumbo-sacral plexus. J. Physiol. (Lond.) 13: 621-772.

Smith, C. L., and M. Hollyday (1983) The development and postnatal organization of motor nuclei in the rat thoracic spinal cord. J. Comp. Neurol. 220: 16-28.

Somjen, G. (1972) Sensory Coding in the Mammalian Nervous System (Neuroscience Series 4), Appleton-Century-Crofts, New York.

Sprague, J. M. (1948) A study of motor cell localization in the spinal cord of the rhesus monkey. Am. J. Anat. 82: 1-26.

Stirling, R. V., and D. Summerbell (1979) The segmentation of axons from the segmental nerve roots to the chick wing. Nature 278: 640642.

Swett, J. S., E. Eldred, and J. S. Buchwald (1970) Somatotopic cordto-muscle relations in efferent innervation of cat gastrocnemius. Am. J. Physiol. 219: 762-766.

Thomas, P. K. (1964) Changes in the endoneurial sheaths of peripheral myelinated nerve fibers during Wallerian degeneration. J. Anat. 98 : 175-182.

Ueyama, R. (1978) The topography of root fibers within the sciatic nerve trunk of the dog. J. Anat. 123: 277-290.

Weeks, O. I., and A. W. English (1985) Compartmentalization of the cat lateral gastrocnemius motor nucleus. J. Comp. Neurol. 235: 255267.

Whitelaw, V., and M. Hollyday (1983) Position-dependent motor innervation of the chick hindlimb following serial and parallel duplications of limb segments. J. Neurosci. 3: 1216-1225.

Wigston, D. J. (1986) Selective innervation of transplanted limb muscle by regenerating motor axons in the axolotl. J. Neurosci. $6: 2757-$ 2763.

Wigston, D. J., and J. R. Sanes (1982) Selective reinnervation of adult mammalian muscle by axons from different segmental levels. Nature 299: 464-467.

Wigston, D. J., and J. R. Sanes (1985) Selective reinnervation of intercostal muscles transplanted from different segmental levels to a common site. J. Neurosci. 5: 1208-1221. 